\title{
Clusters Regularly Interspaced Short Palindromic Repeats Surface-Enhanced Raman Scattering for the detection of the drug resistance gene MacB
}

\author{
yuwan Du ( $\sim 1600759488 @ q q . c o m)$
}

tianjin https://orcid.org/0000-0002-8699-9411

\section{Dianpeng Han}

Ecole du Val-de-Grace Department of Environmental Preparedness and Operations: Ecole du Val-deGrace Departement de la preparation milieux et operationnelle

\section{Zhaoxia An}

Heibei university

Jiang Wang

Tianjin institute of environmental and operation medicine

\section{Zhixian Gao}

Ecole du Val-de-Grace Department of Environmental Preparedness and Operations: Ecole du Val-deGrace Departement de la preparation milieux et operationnelle

\section{Research}

Keywords: CRISPR/dCas9, Surface-Enhanced Raman Scattering, drug resistance gene MacB, gold nanoparticles

Posted Date: September 28th, 2021

DOl: https://doi.org/10.21203/rs.3.rs-934185/v1

License: (c) (1) This work is licensed under a Creative Commons Attribution 4.0 International License. Read Full License 
Clusters Regularly Interspaced Short Palindromic Repeats Surface-Enhanced Raman Scattering for the detection of the drug resistance gene $\mathrm{MacB}$

Yuwan Du ${ }^{\mathrm{a}}$, Dianpeng Han ${ }^{\mathrm{a}}$,Zhaoxia An ${ }^{\mathrm{b}}$,Jiang Wang ${ }^{\mathrm{a}}$, Zhixian Gao ${ }^{\mathrm{a}^{*}}$

${ }^{a}$ Tianjin Key Laboratory of Risk Assessment and Control Technology for Environment and Food Safety, Tianjin Institute of Environment and Operational Medicine, Tianjin300050, P. R. China;

${ }^{\mathrm{b}}$ Public health and preventive medicine, Hebei University, Hebei, 71000, China;

* Corresponding Authors: Zhixian Gao Email: gaozhx@163.com; 


\section{$1 \quad$ Abstract}

2 Antibiotics have brought many benefits to public health systems

3 worldwide since they were first used in the last century, yet with the

4 overuse of antibiotics in clinical care and livestock farming, new public

5 health issues have arisen.In our previous experiments, we found that the

6 MacB gene in bovine raw milk ranked first among many drug resistance

7 genes.In this paper, we present a strategy for the highly sensitive

8 detection of the drug resistance gene MacB by combining clusters

9 regularly interspaced short palindromic repeats (CRISPR) with

10 surface-enhanced Raman scattering (SERS) technology.This strategy

11 integrates the specific gene identification capabilities of the CRISPR

12 system, the ultra-sensitivity of SERS, the chemometric algorithm analysis

13 and the simple separation properties of nanoparticles, eliminating the

14 need for complex steps such as purification and gene amplification,

15 offering the advantages of rapid, ultra-sensitive and highly specific

16 detection, thus enabling more effective tongue safety.

17 Keywords: CRISPR/dCas9; Surface-Enhanced Raman Scattering; drug 18 resistance gene MacB; gold nanoparticles. 
3 husbandry sectors due to their low cost and efficient pharmacokinetic

4 properties ${ }^{1}$. However, overuse of antibiotics not only causes an increase

5 in bacterial resistance, but also results in excessive residues of antibiotics

6 in food of animal origin, as well as leading to cross-transmission of

7 resistance genes in the human-animal-environment, posing a serious risk

8 to public health.Accurate and sensitive detection of drug resistance genes

9 in food is therefore essential for food safety and human health.Currently,

10 the detection of multiple drug resistance genes is usually performed by

11 polymerase chain reaction (PCR), nucleic acid sequence amplification

12 (NASBA), recombinase-based polymerase amplification (RPA),

13 loop-mediated isothermal amplification (LAMP) and aggregation of

14 regularly interspaced short palindromic repeats (CRISPR)-related

15 nucleases (CRISPR/Cas). Although these amplification methods have

16 high sensitivity and specificity, they often have multiple steps, require

17 long reaction times, complex primer designs, multiple reagents and

18 relevant instruments, etc. In addition, they are prone to contamination and

19 false-positive results during the process, which cannot be completely

20 avoided at present. To overcome the limitations of traditional nucleic acid

21 amplification methods ${ }^{2,3}$, we proposed the following strategy, a detection

method based on regularly interspaced clustered short echo repeat 
1 sequences aggregation (CRISPR) combined with surface-enhanced

2 Raman scattering (SERS) technology $y^{4,5}$.

3 In recent years, the CRISPR/Cas system has become a revolutionary

4 tool for targeted genome editing and this technology has great potential

5 for molecular diagnostic applications ${ }^{6,7}$. Nucleic acid detection methods

6 based on this approach have the ability to specifically identify gene

7 sequences, i.e. the gene editing protein in this technology is able to bind

8 to the target gene in a specific manner via a single guide RNA (sgRNA)

9 binding site, meaning that the sgRNA can be easily edited to recognise

10 any target gene sequence ${ }^{8}$. Current applications of this method require

11 sample extraction and amplification of nucleic acids ${ }^{9},{ }^{10},{ }^{11}$ making it

12 challenging to use it more simply and accurately for the detection of

13 bacterial resistance genes ${ }^{12,13}$.

14 Surface-enhanced Raman scattering ${ }^{14}$ has been developed as a

15 sensitive method for the preparation of biosensors, which is an optical 16 sensing technique with molecular fingerprinting, sensitivity at the 17 molecular level and retardation to fluorescence bursts. It has therefore 18 been applied to detect bacterial genes or to detect bacterial genes directly

19 without a label, and a variety of SERS label-free rapid detection of 20 bacteria have been reported ${ }^{15,}{ }^{16}$.The tagged SERS assay has improved 21 the sensitivity and stability of the assay ${ }^{17}, 18$. SERS-based genetic 22 detection methods can achieve accurate classification of 
1 bacteria.Combining the sequence-specific recognition capabilities of

2 CRISPR/Cas-based systems with the highly sensitive synergy of SERS

3 will provide a simple, rapid and sensitive method to detect bacterial drug

4 resistance genes.Firstly, the CRISPR/Cas-based systems are mainly

5 detected using fluorescence techniques, which are easy to observe ${ }^{19,20}$.

6 Moreover, CRISPR/Cas system-mediated SERS sensing methods are less

7 studied, where the dCas9/sgRNA ribonucleoprotein (RNP) complex was

8 designed as a highly specific and efficient targeting DNA probe that acts

9 similarly as a DNA-targeting and labelling antibody.In this study, RNP

10 complexes were combined with gold nanoparticles to form detection

11 probes reacting with the bacterial drug resistance gene MacB gene.

12 Enhanced Raman spectroscopy signals were obtained from the captured

13 detection probes following the incorporation of Raman reporter

14 molecules. This strategy allows us to obtain the ability to detect fM levels

15 without nucleic acid amplification. The method can also be extended to

16 detect a wide range of genes, providing a new detection method in food

17 safety testing.

\section{Principles of CRISPR-SERS analysis}

In the principle of the CRISPR-SERS technology combination, genomic DNA can be used directly without the need for complex purification and amplification steps for effective detection of bacterial 
1 resistance. Probes formed by gold nanoparticles in complex with RNP

2 and DNA from macrolide antibiotic resistance genomic MacB are mixed

3 and incubated. Since the dCas9/sgRNA complex in the CRISPR system is

4 able to scan the entire genome and is able to capture and bind

5 complementary target gene sequences through specific $\operatorname{sites}^{21,22}$, the

6 Raman reporter molecule methylene blue (MB) was later added to the

7 mix and embedded in the target DNA. MB is a $638 \mathrm{~nm}$ excitation source

8 and a sensitive Raman reporter molecule used for double-stranded DNA

9 staining. sensitive Raman reporter molecule, which has been successfully

10 applied for DNA quantification in solutions, gels, real-time PCR and cell

11 staining due to its significant increase in fluorescence emission upon

12 interaction with double-stranded DNA 23, 24. Measurements were

13 completed and Raman spectral signals were obtained by centrifugation of

14 gold nanoparticles bound to the drug-resistant MacB gene following the

15 formation of a probe in complex with RNP.

\section{Materials and methods}

\subsection{Materials and instruments}

$\mathrm{HNO}_{3} / \mathrm{HCl}$, tetrachloroalloy acid $\left(\mathrm{HAuCl}_{4}\right)$ was purchased from Shanghai Aladdin Biochemical Technology Co. Ltd.; 1-ethyl-(3-dimethylaminopropyl)carbodiimide

(EDC) and N-hydroxysuccinimide (NHS) were purchased from Sigma-Aldrich, 
1 nickel chloride $\left(\mathrm{NiCl}_{2}\right)$ and NHS. Ltd.; MOPs

2 (3-(N-morpholino)propanesulfonic acid) were purchased from Beijing

3 Puyihua Technology Co. Ltd.; gel nucleic acid stain and methylene blue

4 (MB) and all related buffers were purchased from Beijing Bajie

5 Biotechnology Co. Dalian TaKaRa Company); SDS-PAGE precast gel

$6 \quad(8 \%)$ was purchased from Beijing Biyuntian Biotechnology Co.

7 TEM images were obtained using the JEM $2100 \mathrm{f}$ microscope of

8 Nippon electronics, which operates at 200kV. Mapping analysis was

9 obtained using Oxford X-MAX 80TLE instrument in Oxford, UK. XPS

10 analysis was obtained using semefi $250 \mathrm{Xi}$ and monochromatic $\mathrm{Al}$ target

11 test (energy 1486.6ev). Raman spectra were obtained by Raman

12 spectrometer (Horiba xplora, France) and excitation source $\lambda=638 \mathrm{~nm}$,

$1330 \mathrm{MW}$ neon laser, through $100 \times$ The objective lens is focused on the

14 sample, and the Raman spectrum of the silicon wafer at $520.7 \mathrm{~cm}^{-1}$ is used

15 to calibrate the instrument.

\subsection{Synthesis of Au/NTA-Ni ${ }^{2+}$ nanoparticles}

Due to the ability of the precious metal shell to produce a SERS response, a large number of SERS hotspots can be formed when the metal is aggregated ${ }^{25}$; the surface wrapped in precious metal is easily modified with various biomolecules, indicating that nanoparticles can be effectively used in a variety of fields such as bioassay.In this paper we 
1 synthesised gold nanoparticles. First, all glassware was soaked in freshly

2 prepared aqua regia $\left(\mathrm{HNO}_{3} / \mathrm{HCl}=1: 3\right)$ and thoroughly rinsed and dried in

3 ultrapure water. AuNPs were prepared by sodium citrate reduction

4 according to a previous report ${ }^{26}: 100 \mathrm{~mL}$ of $0.01 \%(\mathrm{w} / \mathrm{v})$ chloroauric acid

$5\left(\mathrm{HAuCl}_{4}\right)$ was added to a $250 \mathrm{~mL}$ round bottom flask, then the solution

6 was heated to boiling at $140^{\circ} \mathrm{C}, 400 \mathrm{rpm}$ and a $1 \%$ sodium citrate

7 solution $\left(\mathrm{NasC}_{6} \mathrm{HsO}_{7}-2 \mathrm{H}_{2} \mathrm{O}\right)$ was quickly added.The colour of the

8 solution changed from light yellow to purple, then to dark red and finally

9 to burgundy within $5 \mathrm{~min}$. After continuing to heat for $15 \mathrm{~min}$, the solution

10 was cooled to room temperature and the prepared solution was stored in a

11 brown mill-mouth flask at $4{ }^{\circ} \mathrm{C}$ for backup. The prepared $\mathrm{Au}$

12 nanoparticles were washed 3 times with ultrapure water, dispersed in

13 ultrapure water with MPA, adjusted the $\mathrm{pH}$ to 9-11 with alkali, stirred

14 overnight at room temperature, centrifuged and separated, washed 3-5

15 times with PBS buffer and finally dispersed in PBS buffer to obtain the

16 surface carboxylated Au/MPA nanoparticles(The lye solution is $0.1 \mathrm{~mol} / \mathrm{L}$

$17 \mathrm{NaOH}$ or $\mathrm{KOH}$ solution; the mass ratio of $\mathrm{Au}$ nanoparticles to MPA is 1 :

183 to 20).EDC was added to the surface carboxylated $\mathrm{Au}$ nanoparticles

19 (Au/MPA) solution made and stirred for $0.5 \sim 1 \mathrm{~h}$; then NHS was added

20 and stirred for $0.5 \sim 1.5 \mathrm{~h}$; then NTA dissolved in PBS buffer was added

21 and stirred overnight at room temperature, the reaction was completed

22 with magnetic separation, washed several times with ultrapure water and 
1 dispersed in ultrapure water; finally an excess of $\mathrm{NiCl}_{2}$ solution was

2 added and The reaction was carried out for $2-4 \mathrm{~h}$, washed 3 times with

3 binding buffer, and finally dispersed in binding buffer to obtain

$4 \mathrm{Au} / \mathrm{NTA}-\mathrm{Ni}^{2+}$ nanoparticles $\left(\mathrm{NiCl}_{2}\right.$ solution solubility of $1 \mathrm{M}$; binding

5 buffer containing 50mM MOPs (3-(N-morpholino)propanesulfonic acid),

$6 \mathrm{pH} 7.9$; the mass ratio of Au/MPA, EDC, NHS and NTA was $1: 2$ to 10 :

72 to $10: 0.1$ to 5$)$.

\subsection{Approach to CRISPR-SERE technology}

For dCas9/sgRNA mobility shift analysis experiments, RNP complexes were constructed by incubating 10ul of sgRNA (10uM) and 40ul of dCas9 protein $(5 \mathrm{uM})$ in $50 \mathrm{ul}$ of PBS for $10 \mathrm{~min}$ at room temperature. The incubated RNP complexes were mixed with PCR products $(50 \mathrm{ng} / \mathrm{ul})$. and incubated in $10 \mathrm{ul}$ of $1 \times$ FastDigest buffer for $60 \mathrm{~min}$ at $37^{\circ} \mathrm{C}$. After adding $6 \times \mathrm{DNA}$ loading buffer and mixing, polyacrylamide gel electrophoresis (PAGE) was performed and visualised using the Gel DocTM imaging system.

To construct Au-dCas9/sgRNA probes, we collected the modified gold nanoparticle solutions by centrifugation and then incubated Au-NTA-Ni ${ }^{2+}$ with $60 \mathrm{ul}$ of dCas9/sgRNA in double buffer containing $100 \mathrm{mMPBS}$ and $30 \mathrm{mM}$ HEPES for $30 \mathrm{~min}$ and washed away the 
1 unbound RNP complexes with PBS. The formation of Au-dCas9/sgRNA

2 probes was verified by mapping of TEM.

3 The Au-dCas9/sgRNA probe was bound to the target gene sequence

4 by incubating 10ul of Au-dCas9/sgRNA probe with 100ng of PCR

5 product in $10 \mathrm{ul}$ of $1 \times$ FastDigest buffer for $60 \mathrm{~min}$ at $37^{\circ} \mathrm{C}$.After

6 completion of the reaction the bound Au-dCas9/sgRNA-DNA was

7 collected by centrifugation, mixed with $6 \times$ DNA loading buffer, subjected

8 to polyacrylamide gel electrophoresis (PAGE) and visualised by gel

9 DocTM imaging system.

10 SERS Raman spectroscopy signals were obtained for analysis. $60 \mathrm{ul}$

11 of Au-dCas9/sgRNA probe was incubated sequentially with different

12 levels of PCR products in $10 \mathrm{ul}$ of $1 \times$ FastDigest buffer at $37^{\circ} \mathrm{C}$ for 60

13 min. $10 \mathrm{ul}$ of methylene blue (MB) was added and incubated for $10 \mathrm{~min}$.

14 The reaction was collected by centrifugation with the target

$15 \mathrm{Au}-\mathrm{dCas} 9 / \mathrm{sgRNA}$ probes bound to the target gene. The collected 16 Au-dCas9/sgRNA-DNA was dropped onto a glass slide and the Raman 17 spectral signal was monitored.

\section{Assessment of CRISPR-SERS results}

In our preliminary experiments, we found by chance that despite originating from different dairy farms, the abundance of macrolide antibiotics was significantly higher than other types of antibiotics, both in 
1 raw milk and in fresh cow manure, corresponding to a much higher

2 abundance of MacB genes for macrolide antibiotic resistance (Figure S1

3 in the Supporting Information), which may be related to the fact that

4 dairy farming The high use of macrolide antibiotics in veterinary

5 medicine at the stage of Macrolide antibiotics, including erythromycin,

6 diclofenac and tylosin, are a group of 14 to 16-membered lactone rings

7 and different additional sugars that inhibit protein translation by targeting

8 bacterial ribosomes and are often used in livestock farming as feed

9 additives and oral delivery agents. In bacteria, resistance mechanisms

10 against macrolide antibiotics can be mediated by a variety of mechanisms,

11 such as ribosomal methylation modifications, activated efflux pumps and

12 macrolide antibiotic inactivating enzymes in addition to the MacB gene ${ }^{27}$,

$13 \quad 28$. As macrolide antibiotics are common in clinical and animal husbandry

14 settings, if consumers continue to acquire resistance genes from ingested

15 food, resulting in the transfer of bacterial resistance to humans via the

16 "farm-to-table" route, there will be a huge economic loss in dealing with

17 infections with resistant pathogens. The rapid detection of MacB genes in

18 milk is therefore important for controlling the transfer of drug-resistant

19 genes from pathogenic bacteria.

To specifically identify the drug resistance gene MacB, we designed 21 sgRNAs for its sequence (Figure S1 in Supporting Information, Table S1)

and we used polyacrylamide gel electrophoresis to assess the accuracy of 
1 the designed sgRNAs, which could effectively demonstrate the binding of

2 the RNP complex to the target gene. RNP complexes were prepared by

3 mixing the dCas9 protein and sgRNA. The PCR amplification products

4 containing the target gene sequences were obtained from bacterial

5 genomic DNA extracted from milk samples (Table S2 in the Supporting

6 Information) and the results of the electrophoretic mobility of the mixture

7 of PCR product, RNP complex and PCR product are shown in Figure S2

8 in the Supporting Information. It can be learned that the electrophoretic

9 bands of the mixture of RNP complex and PCR product are more upward compared to the PCR product, mainly because the binding of the PCR

11 product to the RNP complex results in slower mobility and therefore not

12 as fast as the electrophoretic band migration rate of the PCR product ${ }^{29}$.

13 This result can indicate that the experimentally designed sgRNA can

14 accurately recognize the target gene sequence, and we learned that the use

15 of dCas9/sgRNA complex does not require cleavage of the MacB gene 16 sequence.

The principle of Au-dCas9/sgRNA binding and target gene sequence detection by SERS is shown in Figure 1. In Au-dCas9/sgRNA binding, 19 gold is coupled to the amino group of nitrogen triacetic acid (NTA), which then chelates $\mathrm{Ni}^{2+}$ to obtain $\mathrm{Au}-\mathrm{NTA}-\mathrm{Ni}^{2+}$. After washing and centrifugation, X-ray photoelectron spectroscopy (XPS) was performed to 22 characterise the Au-NTA-Ni ${ }^{2+}$ peaks (Supporting Infographic S3), which 
1 were fitted with a non-linear least squares procedure to produce an S2p

$2(162.6 \mathrm{eV})$ peak, similar to the S2p binding energy of the Au-S bond,

3 which could indicate that the - $\mathrm{SH}$ of MPA is bound to the gold

4 nanoparticle surface via Au-S. The high-resolution $\mathrm{C} 1 \mathrm{~s}(284.7 \mathrm{eV})$ peak

5 generated by Au-NTA-Ni ${ }^{2+}$ corresponds to the binding ability of $\mathrm{C} 1 \mathrm{~s}$ of

$6-(\mathrm{CH} 2)^{-30,31}$. The Ni2p1/2 $(874.2 \mathrm{eV})$ and Ni2p3/2 $(856.7 \mathrm{eV})$ generated

7 were derived from $\mathrm{Ni}^{2+}$ chelates. xPS results indicate the successful

8 preparation of $\mathrm{Au}-\mathrm{NTA}-\mathrm{Ni}^{2+}$ and we know that the dCas9 protein with a

$96 \times$ His histidine tag is susceptible to binding metal ions during protein purification. We were therefore able to obtain Au-dCas9/sgRNA probes

11 by mixing the prepared Au-NTA-Ni ${ }^{2+}$ with the dCas $9 / \operatorname{sgRNA}$ complex ${ }^{32}$. mapping analysis of the probe was performed (Supporting Infographic S4) and the addition of the dCas $9 / \mathrm{sgRNA}$ complex resulted in the presence of large amounts of $\mathrm{C}, \mathrm{O}, \mathrm{N}$ and $\mathrm{S}$ elements on the surface of the gold. Thus

To confirm the formation of the Au-dCas9/sgRNA probe, a TEM being able to account for the formation of the Au-dCas9/sgRNA probe.

To verify that the Au-dCas9/sgRNA probe specifically recognises the macrolide antibiotic resistance gene $\mathrm{MacB}$, we determined this by comparing the change in polyacrylamide gel electrophoresis bands before and after the addition of the target gene, as shown in Figure 2. After the addition of DNA bound to the Au-dCas9/sgRNA probe, sparser electrophoretic bands could be observed, indicating specific binding of 
1 the Au-dCas9/sgRNA probe to DNA. To be able to capture the Raman

2 spectral signal, we added a methylene blue Raman reporter molecule,

3 which is a dye capable of mosaicking onto the Au-dCas9/sgRNA probe

4 captured by the DNA double strand, as shown in Figure 3. We used the

5 PCR amplification product of the drug resistance gene MacB for the

6 assay. The PCR product (400ng) was reacted with its corresponding

7 Au-dCas9/sgRNA probe in a test tube after adding the Raman dye

8 methylene blue and testing for SERS signal (the SERS image obtained is

9 shown in Supporting Information S5). When no PCR amplification

10 product was added, only Raman spectra with no distinctive spectral peaks

11 could be obtained (black line in Figure 3). Based on these results we can

12 know that this CRISPR-SERS technique can be used for the detection of

13 the drug resistance gene MacB.

14 In the evaluation of CRISPR-SERS technology, we found that the

15 MacB gene in milk required only 50ng to monitor the Raman signal, and

16 the partial least squares regression PLR algorithm was used to regress the

17 Raman spectra for prediction ${ }^{33}$. After baseline correction of the collected

18 Raman spectral data, the Raman spectral detection provides a wide range

19 of spectral information, which not only contains biological information of

20 the detected objects but also a large amount of redundant information. In

21 order to better ensure the correlation between the characteristic peaks of

22 Raman spectra and the content of PCR products, the characteristic range 
1 of all spectral data was selected, which not only reduces the time to build

2 the model but also improves the This not only reduces the time required

3 to build the model but also improves the accuracy of the model. As the

4 quality of the original spectra was improved after intercepting the spectra,

5 there was still invalid information in the spectra. The normalisation

6 pre-processing method was used to eliminate the drift in the spectral

7 measurements, amplify and separate the overlapping information,

8 improve the signal-to-noise ratio and predict the robustness of the model

9 to obtain more valid spectral information.and PLS modelling was

10 performed on the original and pre-processed spectra. For the acquired

11 Raman spectra after PLS algorithm, the prediction set correlation

12 coefficient $\mathrm{Rp}$ was 0.9695 , the root mean square error of the prediction

13 set RMSEP was 11.7263 and Bias was 2.6945, as shown in Figure 4(a).

14 This prediction model shows that the intensity of the Raman spectrum

15 increases when the PCR product added increases, as shown in Figure 4(b).

16 The linear fitted equation in Figure $4(\mathrm{c})$ is $\mathrm{y}=9.2125 \mathrm{x}+543.02, \mathrm{R}^{2}=0.9960$.

17 based on $\mathrm{LOD}=3 \mathrm{Sb} / \mathrm{m}$ (where $\mathrm{Sb}$ is the standard deviation of the SERS

18 response of the blank sample and $\mathrm{m}$ is the slope of the calibration curve).

19 The LOD of the macrolide antibiotic resistant MacB gene in milk was

20 therefore $32.5 \mathrm{ng}$, converting the LOD value to $17.4 \mathrm{fM}$, and the complete

21 SERS data is shown in Figure S6 in the Supporting Information. The

22 sensitivity of the system can therefore be investigated by measuring the 
1 intensity of the Raman spectra obtained at different levels of PCR product

2 at room temperature. Therefore, in this experiment the Raman spectral

3 peak intensities were able to effectively differentiate between the MacB

4 content of drug resistance genes and the Au-dCas9/sgRNA probe was

5 able to specifically identify complementary target sequences, allowing

6 accurate detection of drug resistance genes in the samples.

$7 \quad$ SERS-based nucleic acid biosensors have been widely used and have

8 been applied in various fields due to the hybridization of nucleic acids

9 that can effectively capture and form SERS Raman spectroscopy hotspots,

10 showing great vitality in the biomedical field with high sensitivity ${ }^{34,35}$. In

11 contrast, the CRISPR/Cas system for nucleic acid detection is currently

12 hot and has received great attention from many scholars, with the

13 characteristic of specific identification of target gene sequences ${ }^{36}$. It is

14 based on the advantages of these two technologies that they are combined.

15 We can know that the combination of CRISPR-SERS technology can

16 contribute to a great extent to the accurate and sensitive detection of

17 target gene sequences. Thus in this experiment we were able to reach the

$18 \mathrm{fM}$ level to detect the drug resistance gene $\mathrm{MacB}$, while we did not need

19 to amplify the gene. Overall, our proposed CRISPR-SERS technology

20 can detect the drug resistance gene MacB in milk samples without the

21 need for multiple complex steps such as amplification and purification. 


\section{Conclusions}

2 In conclusion, we demonstrated that simple, rapid and highly

3 sensitive detection of the drug resistance gene MacB is possible in a

4 system based on CRISPR-SERS technology, with detection limits up to

5 the fM level. The dCas9/sgRNA complex in this system is similar to an

6 antibody in conventional immunisation and acts as a targeting material,

7 acquiring Raman spectroscopic information through the Raman reporter

8 molecule MB. Thus, providing a simple and inexpensive way to obtain

9 the marker. We were able to effectively demonstrate the advantage of

10 higher target specificity of this technique compared to conventional

11 nucleic acid amplification methods ${ }^{37,38}$. As the target of dCas9/sgRNA

12 can be designed by targeting sequences, the technique is not limited to

13 detecting the MacB sequence of drug resistance genes. It can be widely

14 applied to many other gene target sequences, such as other bacteria, 15 viruses, etc.

Ethics approval and consent to participate

Not applicable

Consent for publication

19

Not applicable 
1 Availability of data and materials

All data generated or analysed during this study are included in this

3 published article

4 Competing interests

5 The authors declare that they have no competing interests

\section{$6 \quad$ Funding}

Development Project "Key Technology and Product Development for

9 Efficient Identification and Confirmation of Biological and Radioactive Hazards in Food" 2018YFC160025.

Authors' contributions

Conceptualization: Yuwan Du, Dianpeng Han

Validation: Dianpeng Han

Formal analysis: Jiang Wang

Investigation: Zhaoxia An

Resources: Zhaoxia An

Data Curation: Jiang Wang

Writing - Original Draft: Yuwan Du 


\title{
Project administration: Zhixian Gao
}

Funding acquisition: Zhixian Gao

5

\section{Acknowledgements}

\author{
Not applicable
}

\section{References}

1. Yue, H. A.; Ji, Z. B.; Lei, W. B.; Xd, A.; Yw, A.; Yang, X. B.; Glb, C., Sensitive detection of chloramphenicol based on Ag-DNAzyme-mediated signal amplification modulated by DNA/metal ion interaction. Biosensors Bioelectron. 2019, 127, 45-49.

2. Yamamoto, Y., PCR in diagnosis of infection: detection of bacteria in cerebrospinal fluids. Clin. Diagn. Lab. Immunol. 2002, 9 (3), 508-514.

3. Gracias, K. S.; Mckillip, J. L., A review of conventional detection and enumeration methods for pathogenic bacteria in food. Canadian Journal of Microbiology 2004, 50 (11), 883-890.

4. Mayuramart, O.; Nimsamer, P.; Rattanaburi, S.; Chantaravisoot, N.; Payungporn, S., Detection of severe acute respiratory syndrome coronavirus 2 and influenza viruses based on CRISPR-Cas12a. Experimental biology and medicine (Maywood, N.J.) 246 (4), 400-405.

5. Liu, Y.; Xu, H.; Liu, C.; Peng, L.; Khan, H.; Cui, L.; Huang, R.; Wu, C.; Shen, S.; Wang, S., CRISPR-Cas13a Nanomachine Based Simple Technology for Avian Influenza A (H7N9) Virus On-Site Detection. Journal of Biomedical Nanotechnology 2019, 15 (4), 790-798.

6. Shi, K.; Lei, C. Y.; Zhou, N., Application Progress of CRISPR/Cas in Nucleic Acid Detection. Journal of Instrumental Analysis 2018.

7. Fu, X.; Shi, Y.; Peng, F.; Zhou, M.; Zhang, X. B., Exploring the Trans-Cleavage Activity of CRISPR/Cas12a on Gold Nanoparticles for Stable and Sensitive Biosensing. Analytical Chemistry 2021, $93(11)$.

8. Plagens, A.; Tjaden, B.; Hagemann, A.; Randau, L.; Hensel, R., Characterization of the CRISPR/Cas Subtype I-A System of the Hyperthermophilic Crenarchaeon Thermoproteus tenax. J. Bacteriol. 2012, 194 (10), 2491-500.

9. Pardee, K.; Green, A. A.; Takahashi, M. K.; Braff, D.; Lambert, G.; Lee, J. W.; Ferrante, T.; Ma, D.; Donghia, N.; Fan, M., Rapid, Low-Cost Detection of Zika Virus Using Programmable Biomolecular Components. Cell 2016, 1255-1266.

10. Kennedy, E. M.; Cullen, B. R., Bacterial CRISPR/Cas DNA endonucleases: A revolutionary technology that could dramatically impact viral research and treatment. Virology 2015, 479-480, 213-220.

11. Aman, R.; Ali, Z.; Butt, H.; Mahas, A.; Aljedaani, F.; Khan, M. Z.; Ding, S.; Mahfouz, M., RNA virus interference via CRISPR/Cas13a system in plants. Genome Biology 2018, 19 (1), 1. 
12. Miller, J. B.; Zhang, S.; Kos, P.; Xiong, H.; Siegwart, D. J., Non-Viral CRISPR/Cas Gene Editing In Vitro and In Vivo Enabled by Synthetic Nanoparticle Co-Delivery of Cas9 mRNA and sgRNA. Angewandte Chemie International Edition 2017, 129 (4), 1059.

13. Miller, J. B.; Zhang, S.; Kos, P.; Xiong, H.; Zhou, K.; Perelman, S. S.; Zhu, H.; Siegwart, D. J., Non-Viral CRISPR/Cas Gene Editing In Vitro and In Vivo Enabled by Synthetic Nanoparticle Co-Delivery of Cas9 mRNA and sgRNA. Angewandte Chemie 2017.

14. Langer, J.; Aberasturi, D.; Aizpurua, J.; Alvarez-Puebla, R. A.; Liz-Marzán, L., Present and Future of Surface Enhanced Raman Scattering. ACS Nano 2020, 14 (1).

15. Cristina, G. T.; Peter, R.; Frederiek-Maarten, K.; Benjamin, B.; Dmitry, K.; Willem, W.; Skirtach, A. G.; Nico, B., Label-free Raman characterization of bacteria calls for standardized procedures. J. Microbiol. Methods 2018, 151, 69-75.

16. Zhou, X.; Hu, Z.; Yang, D.; Xie, S.; Jiang, Z.; Niessner, R.; Haisch, C.; Zhou, H.; Sun, P., Bacteria Detection: From Powerful SERS to Its Advanced Compatible Techniques. Advanced Science 2020.

17. Yap, L. W.; Chen, H.; Gao, Y.; Petkovic, K.; Liang, Y.; Si, K. J.; Wang, H.; Tang, Z.; Zhu, Y.; Cheng, W., Bifunctional plasmonic-magnetic particles for an enhanced microfluidic SERS immunoassay. Nanoscale 2017, 10.1039.C7NR01511A.

18. Pearson, B.; Wang, P.; Mills, A.; Pang, S.; Mclandsborough, L.; He, L., Innovative sandwich assay with dual optical and SERS sensing mechanisms for bacterial detection. Analytical methods 2017, 9.

19. Taejoon; Kang; Seung; Min; Yoo; Ilsun; Yoon; Sang; Yup; Lee, Patterned Multiplex Pathogen DNA Detection by Au Particle-on-Wire SERS Sensor. Nano Lett. 2010, 10 (4), 1189-1193.

20. Boris; Khlebtsov; Elizaveta; Panfilova; Vitaly; Khanadeev; Nikolai; Khlebtsov, Improved size-tunable synthesis and SERS properties of Au nanostars. Journal of Nanoparticle Research 2014, 16 (10), 2623.

21. Mekler, V.; Minakhin, L.; Severinov, K., Mechanism of duplex DNA destabilization by RNA-guided Cas9 nuclease during target interrogation. Biophys. J. 2017, 112 (21), 315a.

22. Josephs, E. A.; Dewran, K. D.; Fitzgibbon, C. J.; Joshua, M. M.; Gersbach, C. A.; Marszalek, P. E., Structure and specificity of the RNA-guided endonuclease Cas9 during DNA interrogation, target binding and cleavage. Nucleic Acids Res. 2015, (18), 8924-41.

23. Eom, G.; Kim, H.; Hwang, A.; Kang, T.; Kim, B., Detection of Telomerase Activity as Cancer Biomarker Using Nanogap-Rich Au Nanowire SERS Sensor. 2017.

24. Eom, G.; Kim, H.; Hwang, A.; Son, H.; Choi, Y.; Moon, J.; Kim, D.; Lee, M.; Lim, E.; Jeong, J., Nanogap-Rich Au Nanowire SERS Sensor for Ultrasensitive Telomerase Activity Detection: Application to Gastric and Breast Cancer Tissues Diagnosis. Advanced Functional Materials 2017, 27 (37).

25. Li, J. F.; Zhang, Y. J.; Ding, S. Y.; Panneerselvam, R.; Tian, Z. Q., Core-Shell Nanoparticle-Enhanced Raman Spectroscopy. Chemical Reviews 2017, acs.chemrev.6b00596.

26. Ojea-Jime?Nez, I.; Romero, F. M.; Bastu?S, N. G.; Puntes, V., Small Gold Nanoparticles Synthesized with Sodium Citrate and Heavy Water: Insights into the Reaction Mechanism. Journal of Physical Chemistry C 2010, 114 (4), 1800-1804.

27. TLR13 recognizes bacterial 23S rRNA devoid of erythromycin resistance-forming modification. Science 2012, 337 (6098), 1111-1115. 
1 28. Wright, G. D., Bacterial resistance to antibiotics: enzymatic degradation and modification. Adv. 2 Drug Del. Rev. 2005, 57 (10), 1451-1470.

3 29. B, K. G. A.; C, J. O. K.; B, S. G. H. A.; B, H. K. A.; B, T. K. A.; B, E. K. L. A.; B, J. J. A., A facile, 4 rapid and sensitive detection of MRSA using a CRISPR-mediated DNA FISH method, antibody-like 5 dCas9/sgRNA complex. Biosensors Bioelectron. 2017, 95, 67-71.

6 30. Guo, H.; Wang, W.; Zhou, F., Fast and highly selective separation of His-tagged proteins by 7 Ni2+-carrying magnetic core-shell nanoparticles. Applied Physics A 2019, 125 (5), 334.

8 31. Xie, H. Y.; Rui, Z.; Bo, W.; Feng, Y. J.; Jian, H., Fe 304 /Au Core/Shell Nanoparticles Modified with Ni 2+ -Nitrilotriacetic Acid Specific to Histidine-Tagged Proteins. Journal of Physical Chemistry C 2010, 114 (11), 4825-4830.

11 32. Joanna; Legat; Magdalena; Matczuk; Federica; Scaletti; Luigi; Messori; Andrei; Timerbaev, Erratum to: CE Separation and ICP-MS Detection of Gold Nanoparticles and Their Protein Conjugates. Chromatographia 2017.

33. Blanco, M.; Coello, J.; Iturriaga, H.; Maspoch, S.; Chemometrics, J. P. J.; Systems, I. L., NIR calibration in non-linear systems: Different PLS approaches and artificial neural networks. 2000, 50 (1), 75-82.

34. Garcia-Rico, E.; Alvarez-Puebla, R. A.; Guerrini, L., Direct surface-enhanced Raman scattering (SERS) spectroscopy of nucleic acids: from fundamental studies to real-life applications. Chemical Society Reviews 2018, 10.1039.C7CS00809K.

35. Krajczewski, J.; Kudelski, A., Shell-Isolated Nanoparticle-Enhanced Raman Spectroscopy. Frontiers in Chemistry 2019, 7.

36. Broughton, J. P.; Deng, X.; Yu, G.; Fasching, C. L.; Chiu, C. Y., CRISPR-Cas12-based detection of SARS-CoV-2. Nat. Biotechnol. 2020.

37. Lee, S.; Park, Y. J.; Park, K. G.; Jekarl, D. W.; Chae, H.; Yoo, J. K.; Seo, S. W.; Choi, J. E.; Lim, J. H.; Heo, S. M., Comparative Evaluation of Three Chromogenic Media Combined with Broth Enrichment and the Real-Time PCR-Based Xpert MRSA Assay for Screening of Methicillin-Resistant Staphylococcus aureus in Nasal Swabs. Annals of Laboratory Medicine 2013, 33 (4). 38. Sherlock, O.; Dolan, A.; Humphreys, H., MRSA screening: can one swab be used for both culture and rapid testing? An evaluation of chromogenic culture and subsequent Hain GenoQuick PCR amplification/detection. Clin. Microbiol. Infect. 2010, 16 (7). 


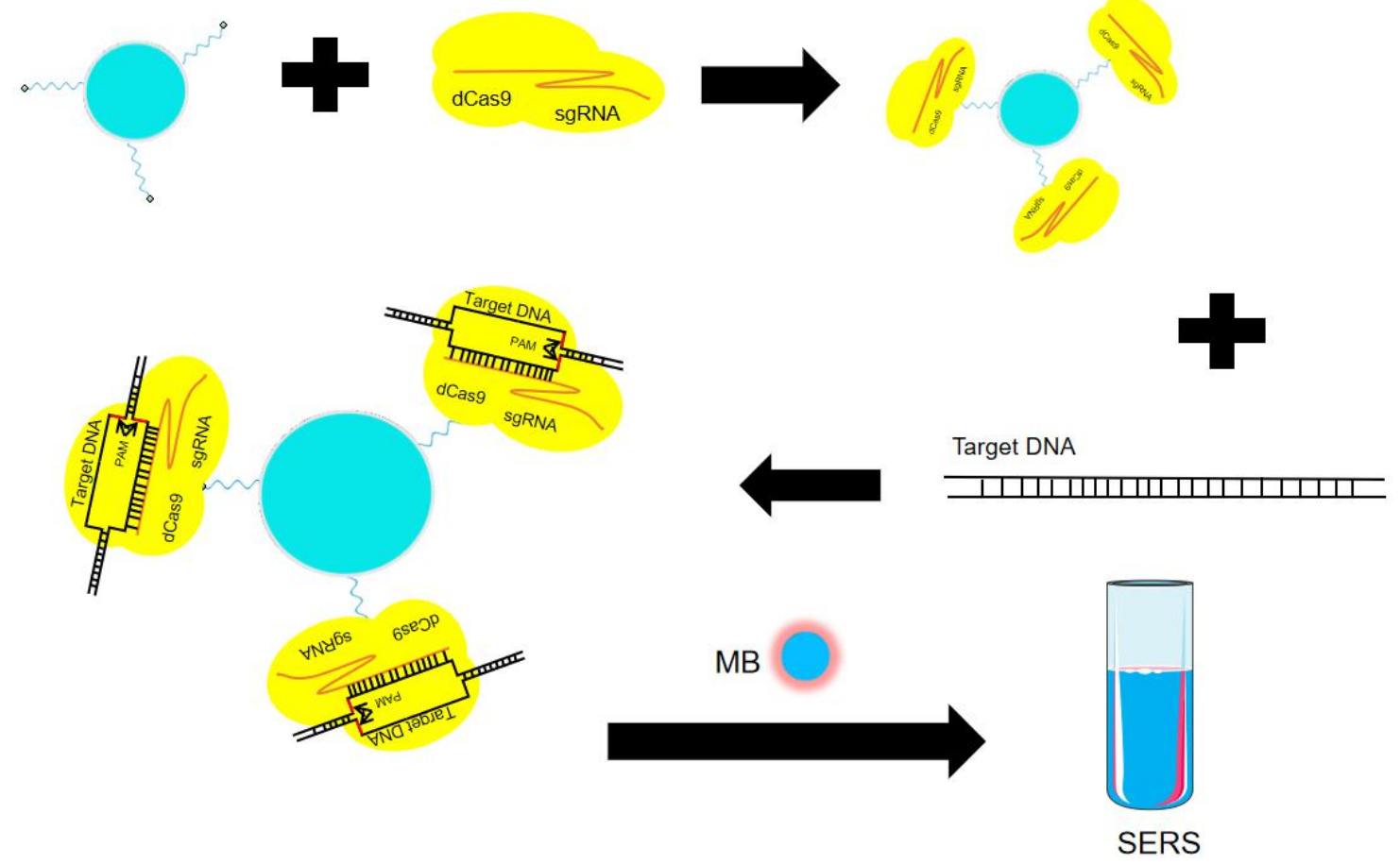

Fig1. Binding of Au-dCas9/sgRNA and detection of target gene sequences by SERS. 


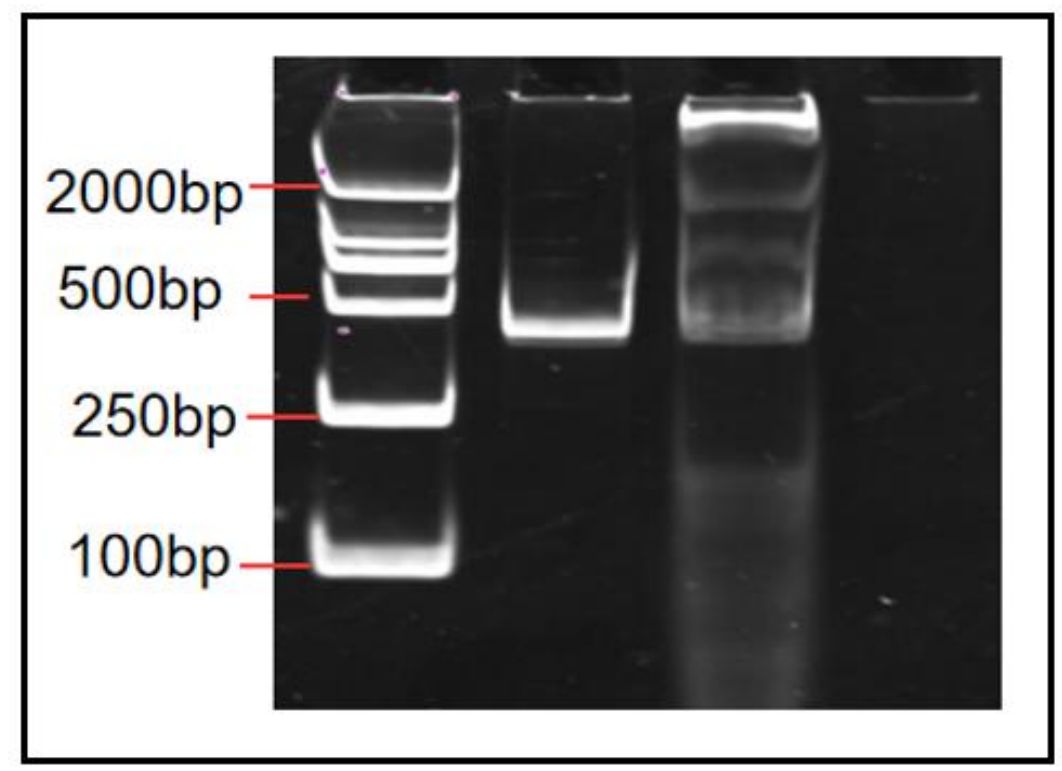

Fig2. polyacrylamide gel electrophoresis images of PCR amplification products before and after reaction with $\mathrm{Au}-\mathrm{dCas} 9 / \mathrm{gRNA}$ probe (line1: PCR product; line2: mixture of PCR product and Au-dCas9/sgRNA probe). 


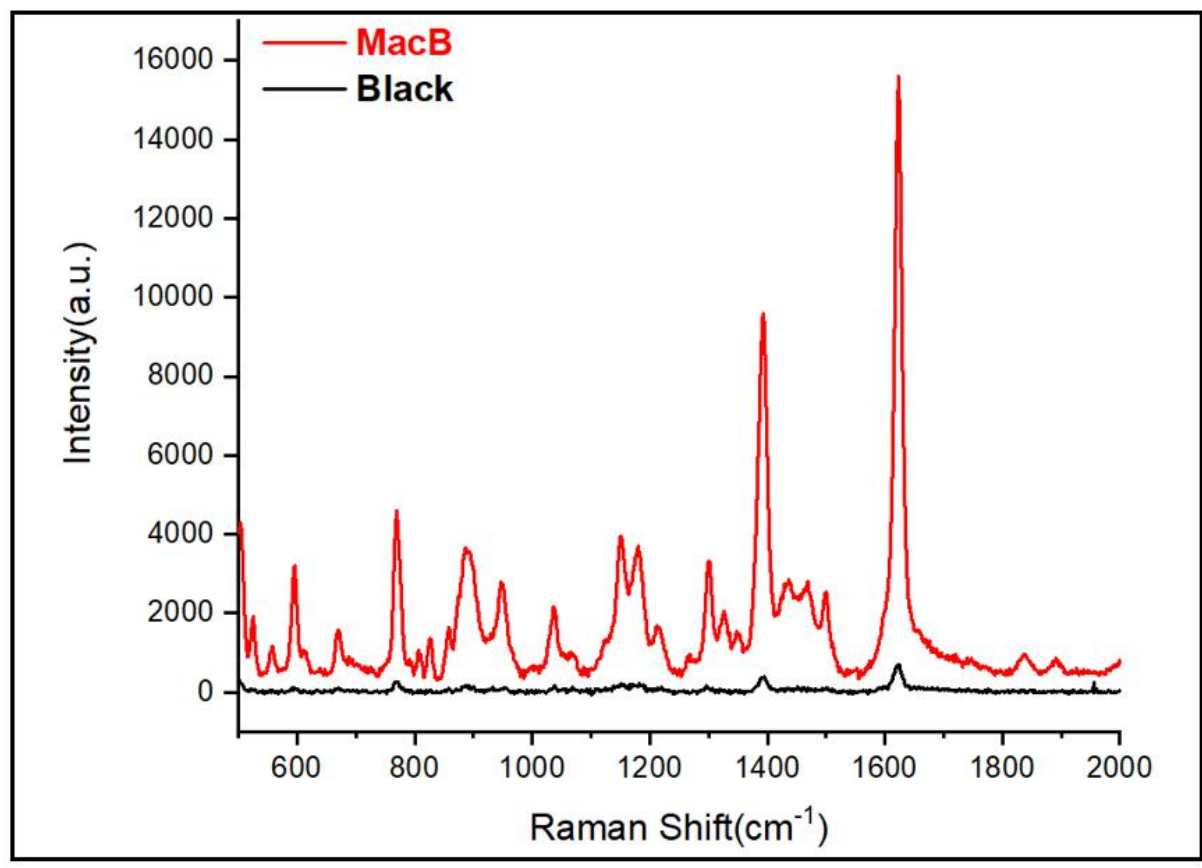

Fig3. Analysis of the PCR amplification product (400ng) by CRISPRP-SER technology. 


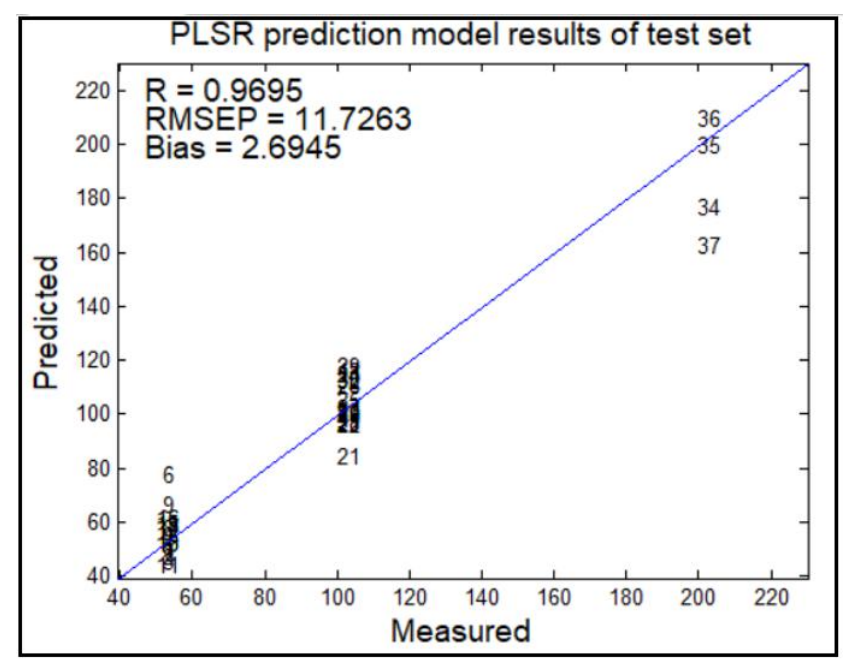

(a)

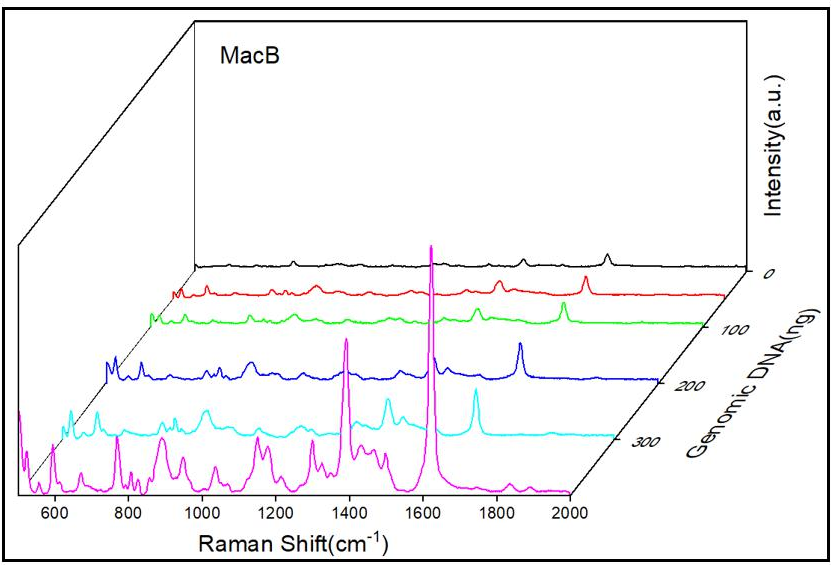

(b)

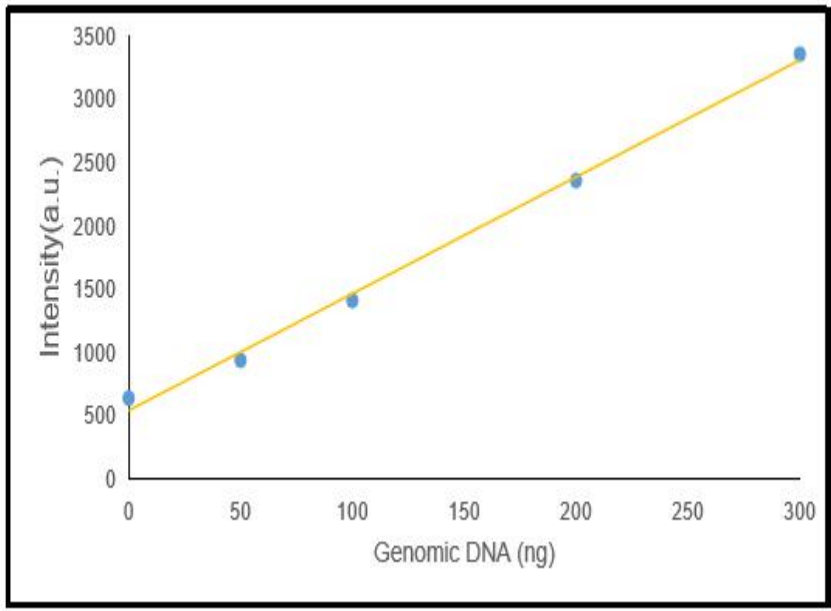

(c)

Fig.4. (a) Results of the PLR prediction model. (b) DNA content of different MacB genomes versus SERS spectra obtained from Au-dcas9/gRNA probe reactions. (c) $1621 \mathrm{~cm}^{-1}$ intensity versus the amount of genomic DNA. 


\section{Supplementary Files}

This is a list of supplementary files associated with this preprint. Click to download.

- GraphicalAbstract0923.pdf

- Supportinformation.pdf 